\title{
$W(b)$-Clefts im Deutschen und Englischen: eine quantitative Untersuchung auf Grundlage des Europarl-Korpus ${ }^{1}$
}

\begin{abstract}
Dieser Beitrag beschäftigt sich mit einem Vergleich der englischen $w h$-Clefts und deren Entsprechungen im Deutschen, den ,Sperrsätzen' oder, $w$-Clefts'. Auf Grundlage einer umfangreichen Korpusstudie werden zunächst Unterschiede in der Verteilung bestimmter $w / h$-Cleftsatztypen ermittelt. Ein generelles quantitatives Übergewicht der englischen $w$-Clefts gegenüber den deutschen $w$-Clefts wird mit der flexibleren Wortstellung des Deutschen in Verbindung gebracht. Spezifisch werden die beobachteten Asymmetrien durch Unterschiede in der Möglichkeit der Erfüllung bestimmter struktureller Bedingungen erklärt. Vier Motivationen für die Bildung von Cleftsätzen werden identifiziert: (i) lineare Synchronisierung von Informationsstruktur und Syntax, (ii) strukturelle Trennung von Quaestio (= im Diskurs gegebener Frage) und Responsio (= Antwort auf die Quaestio), (iii) Trennung von propositionalem Gehalt und Äußerungskommentar (,Ebenentrennung) und (iv) Rechtslastigkeit (Behaghels, Gesetz der wachsenden Glieder). Während all diese Faktoren die Bildung von wh-Cleftsätzen im Englischen zu begünstigen scheinen, sind deutsche $w$-Clefts meist durch den in (ii) genannten Faktor motiviert. Die anderen Motivationen führen seltener zur Bildung von $w$-Cleftsätzen als im Englischen, da die entsprechenden strukturellen Effekte auch ohne Cleftsatzbildung - z.B. in einem kanonischen Verbzweitsatz - erzielt werden können.
\end{abstract}

\section{Einleitung}

Sätze wie (1) und (2) werden in der englischsprachigen Literatur gewöhnlich als ,pseudo-clefts' oder, wh-clefts' bezeichnet (siehe z.B. Akmajian 1970; Prince 1978; Huddleston/Pullum 2002), in der germanistischen Tradition als ,unechte Spaltsätze‘ oder ,Sperrsätze` (vgl. Altmann 1981, 2009; Duden 2009).

Dieser Beitrag enthält Forschungsergebnisse, die im Rahmen des DFG-Projektes „Umfassende Bestandsaufnahme, Beschreibung und Erklärung wesentlicher Kontraste zwischen den Strukturen des Englischen und des Deutschen“ (Ko 497-11/1) erarbeitet wurden. Wir danken der DFG für die Finanzierung dieses Projekts. An der Erhebung und Annotation der hier verwendeten Daten haben neben den Autoren Olga Barsukova, Gerome Bochmann und Marie Schneider mitgewirkt. Wir sind mehreren Kolleginnen und Kollegen für wertvolle Diskussionsbeiträge und Kommentare zu diesem Beitrag zu Dank verpflichtet, insbesondere verschiedenen Teilnehmern der 47. Jahrestagung des IDS. Wir möchten außerdem zwei anonymen Gutachter/inne/ $\mathrm{n}$ und den Herausgebern dieses Bandes für wertvolle Kommentare und Hinweise danken. 
(1) What we need is decentralisation and the Rule of Law. (EPEG-6/De 321541)2

(2) Was wir brauchen, ist Dezentralisierung und die Herrschaft des Rechts.

Der Einfachheit und Einheitlichkeit halber werden wir im Folgenden die deutschen Sperrsätze analog zu den englischen $w h$-Clefts , w-Clefts ${ }^{6}$ nennen (vgl. auch Fischer 2009). Als Generalisierung über die beiden Cleftsatztypen werden wir,$w / h$-Cleft ${ }^{6}$ verwenden.

Englische $w h$-Clefts und deutsche $w$-Clefts sind sich strukturell sehr ähnlich, unterscheiden sich aber erheblich in ihrer Distribution. Da ihre Verwendung (überwiegend) diskurspragmatisch motiviert ist, stellen sie die kontrastive Linguistik vor nicht-triviale Schwierigkeiten. Eine zentrale Aufgabe der kontrastiven Linguistik besteht zweifellos darin, Sprachen im Hinblick auf ihren ,Möglichkeitsraum' zu vergleichen. Im Falle teilweise oder ganz pragmatisch konditionierter Strukturen stellt sich allerdings auch die Frage, in welcher Weise die vorhandenen Möglichkeiten tatsächlich ausgeschöpft werden.

In diesem Beitrag stellen wir eine auf Grundlage des Europarl-Korpus (vgl. Koehn 2005 und Abschnitt 2) durchgeführte quantitative Studie vor, in der wir die Distribution von $w / h$-Clefts im Englischen und Deutschen vergleichen. Wir identifizieren vier ,Motivationen` für die Verwendung von $w h$-Clefts im Englischen, von denen nur eine auch im Deutschen die Bildung von $w$-Clefts systematisch begünstigt. Deutsche $w$-Clefts sind meist durch eine paradigmatische Beziehung der Cleftkonstituente zu einem Element im Diskurskontext motiviert (z.B. Kontrast). Im Englischen erfüllen whClefts teilweise eine rein syntagmatische oder auch satzsemantische Funktion. Letztlich lassen sich die beobachteten quantitativen Unterschiede durch die ,Ökologie‘ der beiden untersuchten Systeme erklären. Das Deutsche verfügt über mehr strukturelle Alternativen zur $w / h$-Cleftsatzbildung als das Englische, wo die Konstruktion ihre ursprünglich diskurspragmatische Funktion teilweise einzubüßen scheint und zunehmend als ,allgemeine Restrukturierungsmaßnahme' verwendet wird.

Nach einigen methodischen Anmerkungen in Abschnitt 2 fassen wir in Abschnitt 3 zunächst die ,Standardbedingungen' für die Verwendung von $w / h$-Clefts zusammen. In Abschnitt 4 werden (verschiedene Typen von) $w / h$ Clefts im Englischen und Deutschen quantitativ miteinander verglichen. Die ermittelten Unterschiede werden in Abschnitt 5 mit funktionalen Motivationen für die Verwendung von $w / h$-Clefts im Deutschen und Englischen in Verbindung gebracht. Abschnitt 6 enthält einige abschließende Bemerkungen.

2 Das Kürzel ,EPEG-6` steht für ,EuroParl English-German, Version 6`. Das Kürzel „De` gibt die Originalsprache an, hier Deutsch; siehe Koehn (2005) und Abschnitt 2 für Informationen über das Korpus. 


\section{Anmerkungen zur Korpusstudie}

Das Europarl-Korpus (Version 6) setzt sich aus Protokollen des Europäischen Parlaments zusammen, die zwischen April 1996 und Dezember 2010 erstellt wurden (vgl. Koehn 2005). Momentan stehen die Protokolle (in unterschiedlichem Umfang) in 21 Sprachen zur Verfügung. Die deutsch-englische Komponente des Europarl-Korpus enthält über 1.700.000 Sätze (jeweils etwa 50 Millionen Wörter). Jedes Teilkorpus enthält sowohl originalsprachliche Reden als auch Übersetzungen. Da das Verhältnis von Originalsprache zu Übersetzung 1:22 ist - es gibt 23 offizielle Sprachen in der EU -, überwiegt naturgemäß der übersetzungssprachliche Anteil in jedem Teilkorpus (wenn auch nicht im Verhältnis 1:22, da einige Originalsprachen deutlich mehr Raum einnehmen als andere; so ist der originalsprachliche Anteil des Deutschen z.B. wesentlich höher als der des Litauischen).

Ein Vorteil dieses Korpusdesigns besteht darin, dass es einen (weitgehend) ,symmetrischen' Sprachvergleich ermöglicht. Beim Vergleich zweier Sprachen liegen in den meisten Fällen Übersetzungen aus einer dritten Sprache zugrunde. In aller Regel weisen die Übersetzungen eine exzellente Qualität auf und es ist aus den Beispielen alleine nicht ersichtlich, ob es sich um originalsprachliches oder übersetztes Material handelt. Dennoch müssen wir uns selbstverständlich darüber im Klaren sein, dass wir, indem wir (überwiegend) Übersetzungen als Datengrundlage verwenden, letztlich zwischensprachliche Entsprechungen vergleichen, die durch Übersetzer hergestellt wurden. Das hat aber wiederum den positiven Effekt, dass wir die bilinguale Kompetenz der jeweiligen Übersetzer dazu nutzen können, Vergleichbarkeit herzustellen. Während die Frage der zwischensprachlichen Vergleichbarkeit in der Sprachtypologie ein bisher ungelöstes Problem darstellt (vgl. Haspelmath 2010), kann sich eine kontrastive, auf Übersetzungen beruhende Studie darauf berufen, dass das, Vergleichbarkeitspostulat ${ }^{\circ}$ empirisch abgesichert ist. Johansson (2000, S. 5) schreibt dazu: ,[t] the use of multilingual corpora, with a variety of texts and a range of translators represented, increases the validity and reliability of the comparison. It can indeed be regarded as the systematic exploitation of the bilingual intuition of translators [...]“ (vgl. auch Gast im Erscheinen).

Ein weiterer Vorbehalt der hier vorgestellten Untersuchung betrifft das Thema ,Registervielfalt'. Das Korpus ist auf das Genre der politischen Rede beschränkt. Insofern gelten alle in dieser Studie gemachten Generalisierungen nur für dieses Genre. Während eine Untersuchung vielfältigerer Registertypen prinzipiell wünschenswert erscheint, hat die Beschränkung auf ein klar definiertes Genre wiederum einen methodischen Vorteil: ${ }^{3}$ Sie erspart uns die Unwägbarkeiten der Definition verschiedener Registertypen und

Ein spezifischer Vorteil des Genres ,politische Rede“ besteht übrigens darin, dass Cleftsätze hier generell verhältnismäßig häufig zu sein scheinen. 
der Erstellung von - idealerweise repräsentativen - Stichproben (vgl. z.B. Biber 1993). Unsere Studie ist also in ihrem Umfang beschränkt, insofern als sie letztlich nur Aussagen über das Genre ,politische Rede' macht, bewegt sich dafür aber auf methodisch sicherem Terrain. Ein Vergleich unserer Ergebnisse mit spezifischen, ebenfalls klar umrissenen anderen Genres könnte sicherlich interessante Ergebnisse liefern. Momentan kann eine solche Untersuchung allerdings nicht auf Grundlage von Parallelkorpora durchgeführt werden, da außer dem Europarl-Korpus ausreichend umfangreiche Parallelkorpora für das Deutsche und Englische nicht verfügbar sind.

Für unseren Vergleich der $w / h$-Clefts im Englischen und Deutschen haben wir zunächst (durch Verwendung von regulären Ausdrücken und nach manueller Filterung) 800 Beispiele aus dem Korpus extrahiert, die sich nach einer zweiten Durchsicht auf 736 (dtsch.) bzw. 742 (engl.) reduzierten. Die meisten dieser später aussortierten Beispiele waren nicht-triviale Grenzfälle zwischen ,echten' $w / h$-Clefts und strukturell identischen prädikativen Konstruktionen (vgl. Abschnitt 4). Wir werden diese Stichproben im Folgenden (approximativ) als ,740er-Stichproben' bezeichnen. Nach erfolgter Filterung wurden alle Cleftsätze im Hinblick auf drei Variablen annotiert: (i) die Kategorie der ,Cleftkonstituente' (d.h. des Komplements der ,höchsten' Kopula; vgl. Abschnitt 4.2), (ii) die syntaktische Funktion des $w / h$-Wortes (Abschnitt 4.3) und (iii) die Art der, anderssprachlichen Entsprechung ${ }^{6}$ - also des Konstruktionstyps, der sich in der jeweiligen Vergleichssprache (Deutsch $\rightarrow$ Englisch, Englisch $\rightarrow$ Deutsch) findet.

Bei der Extraktion (ungefähr) gleich großer Stichproben darf natürlich nicht vergessen werden, dass der Anteil an $w / h$-Clefts in den beiden Korpuskomponenten (deutsch und englisch) nicht identisch ist. Das numerische Verhältnis zwischen englischen und deutschen Clefts lässt sich (approximativ) aus der Variable, anderssprachliche Entsprechung' berechnen. Die von uns ermittelten Werte für diese Variable sind in Tabelle 1 zusammengefasst, wo nur drei Typen der ,anderssprachlichen Entsprechung' unterschieden sind, (i) $w / h$-Cleft, (ii) anderer Cleftsatz und (iii) kein Cleftsatz.

\begin{tabular}{|c|c|c|c|c|}
\hline & $\begin{array}{l}w / h \text {-Cleft in } \\
\text { Vergleichs- } \\
\text { sprache }\end{array}$ & $\begin{array}{l}\text { anderer Cleft- } \\
\text { satz in Ver- } \\
\text { gleichssprache }\end{array}$ & $\begin{array}{l}\text { kein Cleftsatz } \\
\text { in Vergleichs- } \\
\text { sprache }\end{array}$ & gesamt \\
\hline $\begin{array}{l}\text { Englisch } \\
w h \text {-Cleft }\end{array}$ & $17,4 \%$ & $0,4 \%$ & $82,2 \%$ & $100 \%$ \\
\hline $\begin{array}{l}\text { Deutsch } \\
w \text {-Cleft }\end{array}$ & 486 & $2,6 \%$ & $31,9 \%$ & $100 \%$ \\
\hline
\end{tabular}

Tab. 1: Ergebnisse für die Variable, anderssprachliche Entsprechung 
Die erste Zeile gibt an, wie die $w h$-Clefts der englischen 740er-Stichprobe im Deutschen wiedergegeben sind, die zweite Zeile zeigt dasselbe für die deutsche Stichprobe. Nur 17,4\% der englischen $w h$-Clefts entsprechen auch im Deutschen $w$-Clefts, während bei immerhin 65,5\% der deutschen $w$-Clefts auch in der englischen Korpuskomponente ein wh-Cleft auftritt. Die Kategorie ,anderer Cleftsatz' umfasst mit it bzw. es gebildete Cleftssätze - in der Germanistik auch ,Spaltsätze' genannt (vgl. (3b) als Entsprechung von (3a)) - sowie die ,umgekehrten' (reversed) $w / h$-Clefts wie in (4b).

(3) a. Was uns interessiert, ist selbstverständlich das Resultat. (w-Cleft)

b. Obviously, it is the result that interests us. (it-Cleft) (EPEG-6/Fr 792462)

(4) a. Was wir brauchen, ist eine politische Bewertung. (w-Cleft)

b. But political judgement is what is needed. (reversed wh-Cleft) (EPEG-6/It 1309155)

Aus den Zahlen in Tabelle 1 können wir das numerische Verhältnis zwischen englischen und deutschen $w / h$-Clefts im Gesamtkorpus (approximativ) ableiten. Wenn wir die ,anderen' Cleftsatztypen mit den $w / h$-Clefts unter der allgemeineren Kategorie ,Cleftsatz' zusammenfassen, ergibt sich ein Wert von etwa 3,8 zugunsten des Englischen. ${ }^{4}$ Mit anderen Worten: Im englischen Gesamtkorpus kommen fast viermal so viele wh-Cleftsätze vor wie im deutschen Gesamtkorpus. Das müssen wir im Hinterkopf behalten - und wir werden natürlich darauf hinweisen - wenn wir die beiden 740er-Stichproben miteinander vergleichen.

\section{Die Interpretation von $w / h$-Clefts im Diskurs}

Bevor wir in Abschnitt 4 und 5 die Unterschiede zwischen den englischen und deutschen $w / h$-Clefts untersuchen, werden wir in diesem Abschnitt den gemeinsamen Nenner in ihrer Verwendung ermitteln. Wie schon Prince (1978) gezeigt hat, lassen sich relativ robuste notwendige Bedingungen für die Verwendung von $w / h$-Clefts formulieren. Die Ergebnisse unserer Korpusstudie deuten allerdings darauf hin, dass die von Prince (1978) identifi-

\footnotetext{
Dieser Wert ergibt sich aus folgender Überlegung: Die numerischen Verhältnisse zwischen den deutschen und englischen Daten können als Bedingungen aufgefasst werden, die das Gesamtkorpus erfüllen muss: Es muss so beschaffen sein, dass $82,2 \%$ der englischen $w h$ Clefts im Deutschen keine Clefts entsprechen und 31,9\% der deutschen $w$-Clefts im Englischen keine Clefts entsprechen. Diese Bedingungen sind in einem Korpus erfüllt, in dem es 3,8-mal mehr $w / h$-Clefts im Englischen gibt als im Deutschen. Grob gesagt, ist dieses Korpus so beschaffen, dass auf zehn englische $w h$-Cleftsätze jeweils drei deutsche $w$-Cleftsätze kommen, wobei zwei der zehn englischen Clefts auch im Deutschen als Cleft wiedergegeben werden (ca. 20\%, exakt 17,4\%) und zwei der drei deutschen Clefts auch im Englischen einem Cleft entsprechen (ca. 67\%, exakt 65,5\%). Bei genauer Berechnung auf Grundlage der Werte in Tabelle 1 ergibt sich ein Wert von 3,8:1 zugunsten des Englischen.
} 
zierten Bedingungen in bestimmten Fällen - insbesondere im Englischen - teilweise außer Kraft gesetzt sind. Wir interpretieren diese ,nicht-kanonischen' Beispiele gewissermaßen als ,Zweckentfremdung' der $w / h$-Clefts, ähnlich dem aus der Evolutionsforschung geläufigen Vorgang der Exaptation (vgl. Lass 1990 zu Exaptation im Sprachwandel). In solchen Fällen tritt die ,primäre (diskurspragmatische) Funktion der $w / h$-Clefts in den Hintergrund, während ein (meist syntaktischer) ,Nebeneffekt ${ }^{`}$ gewissermaßen ausgeschöpft wird. Wir betrachten daher die von Prince (1978) beschriebenen Bedingungen als, Standardbedingungen' für die Verwendung von $w / h$ Clefts. Es ist gut möglich, dass die ,sekundären', meist syntaktisch motivierten $w / h$-Clefts systematische diachrone Erweiterungen dieser ursprünglich diskurspragmatisch konditionierten Konstruktion darstellen. Aufschlussreich ist dabei vor allem, dass entsprechende Verwendungen fast ausschließlich im Englischen anzutreffen sind. Der Begriff ,Standardbedingung' steht also für die diskurspragmatischen Umstände, unter denen $w / h$-Clefts sowohl im Deutschen als auch im Englischen auftreten, und unter denen sie wahrscheinlich im Englischen (historisch gesehen) zuerst aufgetreten sind.

Prince (1978) hat gezeigt, dass $w / h$-Clefts strengeren diskurspragmatischen Bedingungen unterliegen als it-Clefts. Sie setzen voraus, dass das Material im $w / h$-Satz dem Sprecher aktuell präsent ist. Prince (ebd., S. 888) formuliert die folgende ,discourse condition on $\mathrm{WH}$-clefts“:

(5) Discourse condition on WH-clefts: A WH-cleft will not occur coherently in a discourse if the material inside the (subject) WH-clause does not represent material which the coöperative speaker can assume to be appropriately in the hearer's consciousness at the time of hearing the utterance. (ebd.)

Wie sich aus Princes (1978) detaillierter Analyse ergibt, handelt es sich bei dem ,Material' im $w / h$-Satz um offene Propositionen, die auch als Fragen aufgefasst werden können. Anders ausgedrückt, muss der $w / h$-Satz also Material enthalten, welches einer Frage entspricht, die sich der Hörer nach Einschätzung des Sprechers aktuell stellt. Aus diesem Grund können $w / h$ Clefts diskursinitial nur unter sehr spezifischen Bedingungen verwendet werden. Solche Bedingungen sind z.B. bei wissenschaftlichen Veröffentlichungen gegeben. Der Autor kann voraussetzen, dass ein Leser sich zu Beginn der Lektüre eines Aufsatzes die Frage stellt, worum es in dem Aufsatz geht. Daher kann ein Aufsatz wie in (6) beginnen, während man mit (7) kein Gespräch eröffnen kann (Prince 1978 liefert eine Reihe weiterer aufschlussreicher Beispiele).

(6) What we have set as our goal is the grammatical capacity of children - a part of their linguistic competence. (Klima/Bellugi-Klima 1969, S. 448, zitiert nach Prince 1978, S. 888) 
(7) *Hi! What I've heard about is your work. (Prince 1978, S. 888)

Wir gehen davon aus, dass die Standardbedingungen für die Verwendung von $w / h$-Clefts einfach mithilfe des von Klein/von Stutterheim $(1987,1992)$ vorgeschlagenen Quaestio-Modells bzw. der in Gast/van der Auwera (2011) skizzierten, modifizierten Version dieses Modells beschrieben werden können (vgl. auch Roberts 1996, 2004 und Büring 2003; für die spieltheoretischen Grundlagen dieses Modells siehe Lewis 1969, Hintikka 1973 und Carlsson 1983). Dem Quaestio-Modell entsprechend können Deklarativsätze prinzipiell als Antworten auf (implizite oder explizite) Fragen aufgefasst werden. Die einem Deklarativsatz zugeordnete Frage ist die, Quaestio' dieses Satzes (Büring 2003 spricht von, question under discussion?). Gast/van der Auwera (2011), wie auch Büring (2003), gehen davon aus, dass Quaestios (bzw. ,questions under discussion') hierarchisch geordnet sind. Eine übergeordnete Quaestio wie ,Wie war die Party?` kann z.B. ,aufgespalten' werden in spezifischere Quaestios wie ,Wie war das Essen?' oder,Wie war die Musik?‘. Im Folgenden wird der Begriff, Quaestio' für die spezifische Frage verwendet, auf die ein Satz eine Antwort gibt (Büring 2003: ,current question under discussion'). Die Antwort auf eine Quaestio werden wir analog als ,Responsio' bezeichnen. ${ }^{5}$

Die Interpretation von $w / h$-Clefts kann mit Hilfe des Quaestio-Modells wie folgt beschrieben werden: Der $w / h$-Satz macht die aktuell zur Diskussion stehende Quaestio explizit, und die Cleftkonstituente beinhaltet die dazugehörige Responsio. $W / h$-Clefts können also gewissermaßen als ,integrierte Adjazenzpaare' betrachtet werden - als Frage-Antwort-Paare innerhalb eines einzigen Satzes. Unser Dezentralisierungsbeispiel kann demnach analysiert werden wie in (8) gezeigt:

(8) Was wir brauchen, ist Dezentralisierung.

Quaestio:

(beim Hörer vorausgesetzt): Was brauchen wir?

Responsio:

(Wir brauchen) Dezentralisierung.

Die Diskursbedingungen für $w / h$-Clefts lassen sich im Rahmen des Quaestio-Modells also folgendermaßen zusammenfassen: Die Verwendung von $w / h$-Clefts setzt voraus, dass die in dem $w / h$-Satz ausgedrückte offene Proposition einer Quaestio entspricht, die Teil des Redehintergrundes und folglich dem Hörer aktuell präsent ist bzw. als präsent beim Hörer vorausgesetzt wird.

Philologisch richtiger wäre responsum, Antwort', da responsio im Lateinischen eigentlich einen konfrontativen Sprechakt bezeichnet und eher ,Erwiderung' bedeutet. 


\section{Die Struktur von $w / b$-Clefts}

\subsection{Syntaktische Zweiteilung}

Wie bereits erwähnt wurde, sind deutsche $w$-Clefts und englische $w h$-Clefts aus struktureller Sicht weitgehend parallel. Sie setzen sich aus zwei Teilen zusammen, einem argumentwertigen, durch ein $w$ - bzw. wh-Element eingeleiteten Nebensatz (z.B. Was wir brauchen ...) und einem Prädikat, das aus einer Kopula und ihrem Komplement besteht (... ist Dezentralisierung). Der $w$-Satz im Deutschen wird meist als freier Relativsatz aufgefasst (vgl. z.B. Altmann 2009). In der englischen Literatur ist auch der Standpunkt vertreten worden, dass es sich bei dem $w$ h-Satz um eine indirekte Frage handelt (vgl. Faraci 1971; Ross 2011). Wir werden in dieser Frage keine Stellung beziehen.

$W / h$-Cleftsatzbildung führt also zu einer, syntaktischen Zweiteilung' eines ursprünglich monoklausalen Satzes. Für die Komponenten eines $w / h$ Clefts werden wir die in (9) gezeigten Bezeichnungen verwenden:

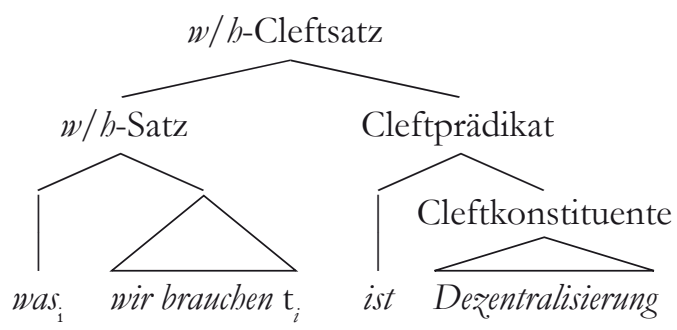

$W / h$-Clefts können als spezifizierende Konstruktionen analysiert werden (vgl. z.B. den Dikken 2009; Fischer 2009). Demnach denotiert der $w / h$-Satz eine Eigenschaft und die Cleftkonstituente fungiert als Argument des die Eigenschaft denotierenden Prädikats (vgl. (10b) als Prädikat-Argument-Struktur von (10a)). Anders ausgedrückt, enthält der $w / b$-Satz eine Variable, dessen Wert durch die Cleftkonstituente spezifiziert wird. Alternativ kann die Semantik von (10a) demnach wie in (10c) dargestellt werden:

(10) a. Was wir brauchen, ist Dezentralisierung.

b. $\lambda x[$ wir brauchen $x]$ (Dezentralisierung)

c. wir brauchen $x, x=$ Dezentralisierung

$W /$ h-Cleftsätze implizieren eine Identitätsaussage auf zwei Ebenen. Zum einen wird - wie in (10c) dargestellt - Identität zwischen der Leerstelle innerhalb des $w / h$-Satzes und der Cleftkonstituente ausgedrückt. Gleichzeitig wird eine Identitätsaussage über den Referenten des $w / h$-Satzes und die Cleftkonstituente gemacht. (10a) kann demnach auch interpretiert werden als ,[NP das, was wir brauchen] ist (identisch mit) Dezentralisierung. Dies ist 
eine Folge davon, dass die Variable innerhalb des $w / h$-Satzes gleichzeitig dessen Referent ist - der $w / h$-Satz lässt sich auch paraphrasieren als ,das $x$, für das gilt: wir brauchen $x^{6}$.

$W / h$-Clefts sind (im Deutschen und Englischen) strukturell nicht von solchen Sätzen zu unterscheiden, in denen einem durch einen $w / b$-Satz repräsentierten Referenten eine Eigenschaft zugewiesen wird, also in prädikativen Verwendungen der Kopula wie in (11a). Hier ist das Prädikat-Argument-Verhältnis gegenüber (10) umgekehrt (vgl. (11b)). Der Äußerung des Adressaten wird die Eigenschaft zugeschrieben, eine Sauerei zu sein (vgl. die extensionale Darstellung in (11c)).

(11) a. Was Sie gerade gesagt haben, ist eine Sauerei.

b. $\lambda p[p$ ist eine Sauerei] (das von Ihnen Gesagte)

c. das von Ihnen Gesagte $\in\{a \mid$ a ist eine Sauerei $\}$

Sätze wie (11) können nicht durch ,Reduktion' in einen äquivalenten einfachen Satz umgeformt werden. Während (10a) mit (12) wegen der oben beschriebenen Identitätsaussage(n) (wahrheitsfunktional) äquivalent ist, kann (11a) nicht durch (13) wiedergegeben werden.

(12) Wir brauchen Dezentralisierung.

(13) ?Sie haben gerade eine Sauerei gesagt.

Der Unterschied wird noch deutlicher sichtbar, wenn man den ,askriptiven Charakter einer prädikativ interpretierten Kopula durch eine entsprechende Prädikation mit halten für explizit macht. Das ist in (11) möglich (vgl. (15)), nicht aber bei (10) (vgl. (14)).

(14) ?Was wir brauchen, halte ich für Dezentralisierung.

(15) Was Sie gerade gesagt haben, halte ich für eine Sauerei.

Die Eigenschaft von $w / h$-Clefts, auf einen einfachen Satz reduzierbar zu sein, ist insofern einer Beschränkung unterworfen, als dass bei prädikatsoder sachverhaltsdenotierenden Cleftkonstituenten (VPn/Prädikate, TPn/ zu-Infinitive) ein Platzhalterverb wie tun oder geschehen im $w$-Satz auftreten kann, das bei der Reduktion getilgt werden muss:

(16) a. Was wir tun müssen, ist, [vp einen Koordinator anbieten] ... (EPEG-6/It 1701062)

b. Wir müssen einen Koordinator anbieten.

(17) a. Was wir heute wirklich tun sollten, ist, [ ${ }_{\text {TP }}$ die Frauen zu ehren ...]. (EPEG-6/Pl 588137)

b. Wir sollten heute wirklich die Frauen ehren ... 
Weiterhin wird in bestimmten Fällen bei der Reduktion auch die Cleftkonstituente verändert. In (17b) fehlt gegenüber (17a) das Infinitiv-zu. Solche ,Inkongruenzen' lassen sich wohl mit Unterschieden zwischen dem kategorialen Status des hier verwendeten $w$-Pronomens - was - und dem der Cleftkonstituente erklären. Während was der Kategorie ,NP' angehört, haben die Cleftkonstituenten in (16) und (17) die Kategorie ,VP' und ,TP'. Idealerweise sollte der Platzhalter innerhalb des $w$-Satzes im Hinblick auf die syntaktische Kategorie mit der Cleftkonstituente übereinstimmen, da, wie oben gezeigt wurde, eine Identitätsaussage gemacht wird. In (16) kann diese Übereinstimmung durch Einfügung von tun hergestellt werden. Die VP [vp was tun] im $w$-Satz kann dann mit der VP [ ${ }_{\mathrm{vp}}$ einen Koordinator anbieten] gleichgesetzt werden. In (17) ist eine solche, Reparatur' nicht möglich, da die Flexion innerhalb des $w$-Satzes nicht ebenfalls durch einen Platzhalter ersetzt werden kann. Es gibt also im $w$-Satz keine Variable der Kategorie ,TP', die mit der Cleftkonstituente gleichgesetzt werden könnte. Eine Reduktion ist daher nur möglich, indem die TP durch Tilgung von $z u$ in eine $\mathrm{VP}$, umgewandelt ${ }^{\natural}$ wird. Letztere kann dann wiederum für [vp was tun] eingesetzt werden.

Eine weitere Einschränkung im Hinblick auf die Möglichkeit der Reduktion von $w / h$-Cleftsätzen hat Ross (1967) als ,Amnestien' bezeichnet, da (scheinbar) Regelverletzungen außer Kraft gesetzt werden. (18a) kann z.B. nicht zu (18b) reduziert werden, da im einfachen Satz eine Anhebungskonstruktion verlangt wird.

a. What I want is that he be punished.

b. *I want that he be punished. (Ross 2011, S. 2)

,Amnestien' wie die in (18) sind etwas anders beschaffen als die in (16) und (17) illustrierten Fälle von ,Nicht-Reduzierbarkeit'. In (18) liegt keine ,kategoriale Inkongruenz' vor, da what sowohl für NPn als auch für CPn stehen kann. Das Problem liegt hier vielmehr in der Subkategorisierung von want, das (in diesem Fall) keine finite Komplementierung erlaubt.

Wir halten fest, dass $w$-Cleftsätze biklausale Strukturen sind, die eine einzige Proposition denotieren und - sofern dies nicht aus syntaktischen Gründen verhindert wird - salva veritate auf einen monoklausalen Satz reduziert werden können.

\subsection{Die syntaktische Kategorie der Cleftkonstituente}

Sowohl das Deutsche als auch das Englische erlauben Cleftsatzbildung mit vier Typen von Cleftkonstituenten (vgl. Prince 1978 für das Englische): (i) Nominalphrasen (NPn), (ii) satzwertige Konstituenten (CPn), (iii) $2 u /$ toInfinitive (TPn) und (iv) Verbalphrasen (VPn). 
- NP-Clefts

(19) What is actually needed however are [ ${ }_{\mathrm{NP}}$ funds and resources]. (EPEG-6/Sp 425590)

(20) Was die Europäische Union jetzt braucht, ist [ NP $_{\mathrm{N}}$ ein schnelleres Wirtschaftswachstum] ... (EPEG-6/Pl 594004)

- CP-Clefts

(21) What this means is $\left[_{\mathrm{CP}}\right.$ that we are in a position to continue the European Union's development in line with what is needed]. (EPEG-6/Ge 281996)

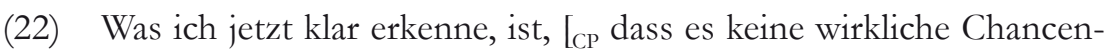
gleichheit in der landwirtschaftlichen Produktion gibt ... ] (EPEG-6/En 595461)

- VP-Clefts

(23) What we have to do is [vp apply the Community acquis and not call other policies into question]. (EPEG-6/Sp 300029)

(24) Was wir nicht können, ist, [vp ein Abkommen schließen, das die WTO dann als nicht kompatibel ablehnt]. (EPEG-6/Po 1543370)

- TP-Clefts

(25) What we do not want is [ TP $_{\text {to }}$ to exempt from transparency obligations the small category of highly specialised financial journalists ...] (EPEG-6/Du 334172)

(26) Was wir wollen, ist ja, [TP den Unfallopferschutz zu verbessern]. (EPEG-6/Ge 1731070)

Die oben angegebenen Kategorien sind jeweils die der Cleftkonstituente. Wie in Abschnitt 4.1 erwähnt, können dabei gewisse ,kategoriale Inkongruenzen' mit den Platzhaltern im $w / h$-Satz auftreten. In (26) z.B. ist die Cleftkonstituente eine TP, während was innerhalb des $w$-Satzes dem Objekt von wollen entspricht und insofern die Kategorie NP oder VP hat.

Im Englischen kommen auch $w / h$-Clefts mit einer Gerundialform (d.h. einer argumentwertigen ing-Form) als Cleftkonstituente vor (vgl. (27)), also einer Kategorie, die es im Deutschen nicht gibt. Hier finden sich unterschiedliche deutsche Entsprechungen, z.B. (nominalisierte) Infinitive oder Nominalisierungen (vgl. die Schaffung in (28) als Entsprechung von creating in (27)).

(27) ... what we primarily need to focus on is creating a system of regulations that enables them to work effectively together.

(EPEG-6/Sv 1262636) 
(28) ... worauf wir uns aber in erster Linie konzentrieren müssen, ist die Schaffung eines Regulierungssystems, das es ihnen ermöglicht, wirksam zusammenzuarbeiten.

Die quantitative Verteilung der fünf oben genannten Kategorien in den 740er-Stichproben ist in Abbildung 1 dargestellt.

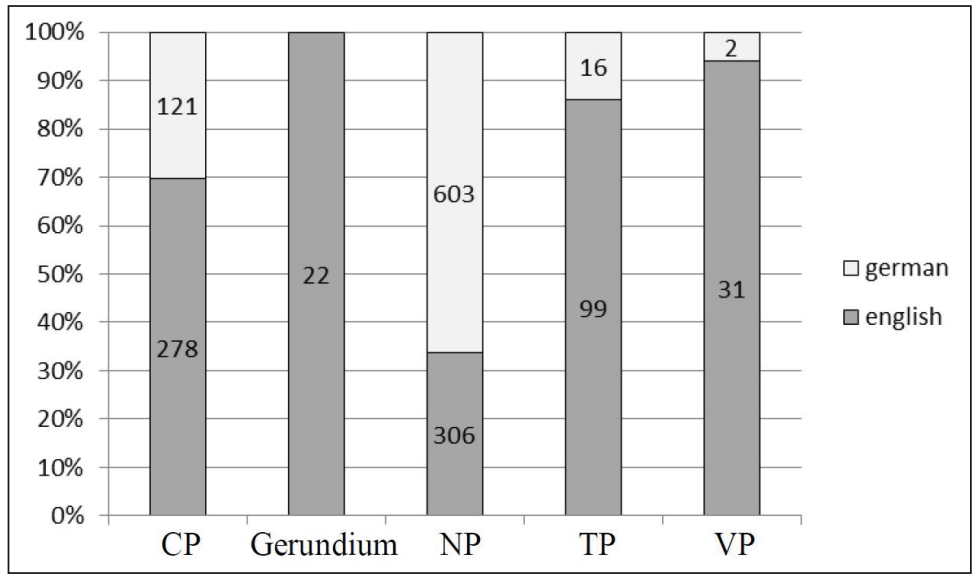

Abb. 1: Die Kategorie der Cleftkonstituente (fünf Typen)

Da es im Deutschen für die Kategorie des Gerundiums keine Entsprechung gibt, haben wir diese Fälle mit to/zu-Infinitiven und VPn unter einer größeren Kategorie zusammengefasst. Was diese Kategorien gemeinsam haben, ist, grob gesagt, dass sie Sachverhaltsbeschreibungen enthalten. Die aus dieser Generalisierung resultierende Verteilung ist in Abbildung 2 gezeigt.

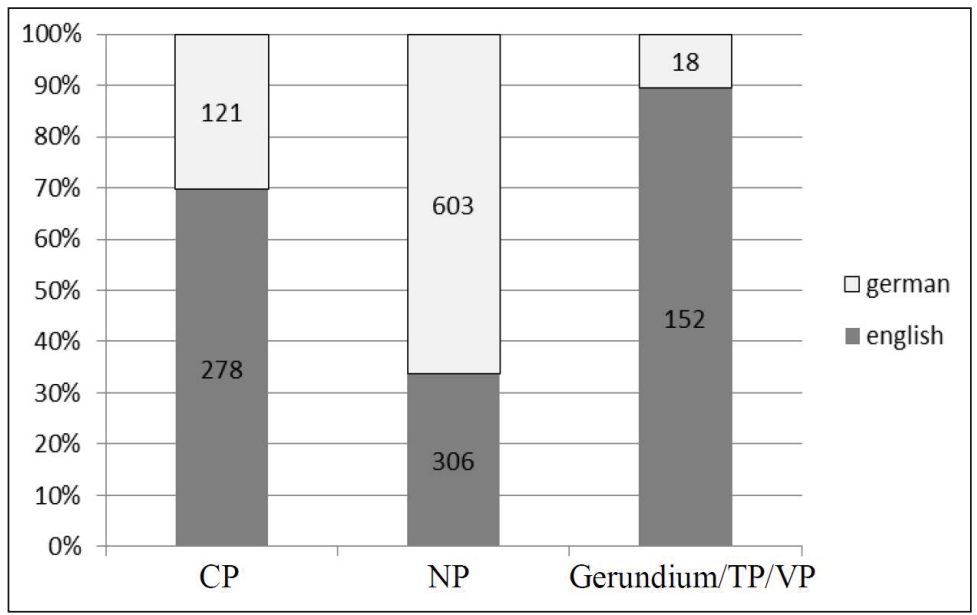

Abb. 2: Kategorie der Cleftkonstituente (3 Typen in Stichprobe) 
Abbildung 1 und 2 bilden jeweils einen Vergleich der beiden 740er-Stichproben ab. Wie in Abschnitt 2 erwähnt wurde, enthält der englische Korpusteil aber etwa 3,8-mal mehr $w / h$-Clefts als der deutsche. Die Gesamtmenge aller Clefts im Korpus verteilt sich (ungefähr) auf ihre Subtypen wie in Abbildung 3 gezeigt, wo die englischen Säulen um den Faktor 3,8 verlängert sind:

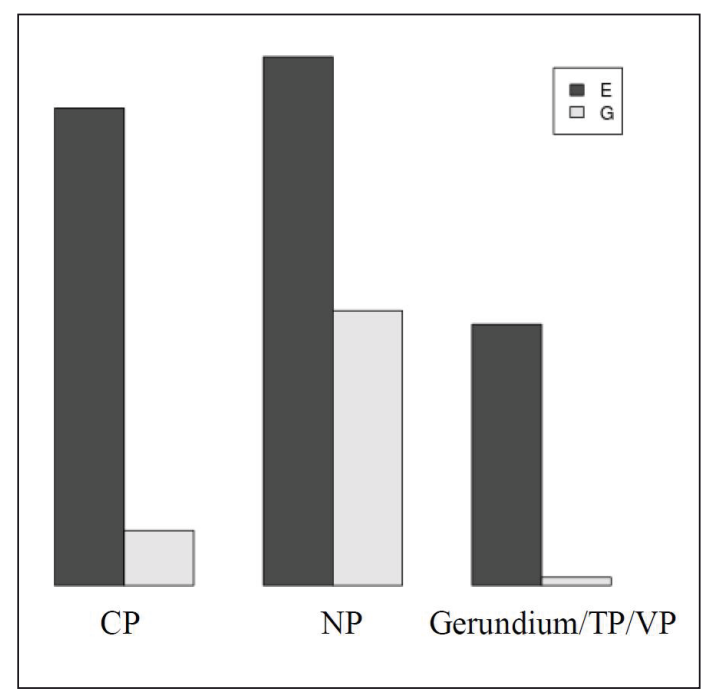

Abb. 3: Kategorie der Cleftkonstituente (3 Typen, projizierte Häufigkeiten)

Die in Abbildung 2 und 3 dargestellten Daten und die sich abzeichnenden Asymmetrien wurden anschließend auf statistische Signifikanz hin überprüft. Die Ergebnisse können sehr gut mithilfe von ,Assoziationsplots' illustriert werden (vgl. Friendly 1992). Abbildung 4 zeigt den Assoziationsplot, der aus den in Abbildung 2 gezeigten Daten generiert wurde.

Abbildung 4 visualisiert die Ergebnisse einer statistischen Analyse einer Kreuztabelle, in der die Variablen ,Sprache' (,deutsch', ,englisch') und ,Kategorie der Cleftkonstituente' (,CP', ,NP', ,Gerundium/TP/VP') gekreuzt wurden (Pearson $\left.\mathrm{X}^{2}=264.42, \mathrm{df}=2, \mathrm{p}<0.001\right)$. Dabei kennzeichnet die gestrichelte Linie den Fall statistischer Unabhängigkeit der gekreuzten Variablen. Die Rechtecke zeigen die Abweichung der jeweiligen Zellenhäufigkeiten von der statistischen Erwartung an. Rechtecke oberhalb dieser Linie zeigen an, dass die jeweilige Kombination von Eigenschaften überzufällig häufig beobachtet wurde. Rechtecke unterhalb der Linie zeigen entsprechend an, dass eine Ausprägungskombination unterzufällig häufig beobachtet wurde. Die Fläche der jeweiligen Rechtecke ist dabei proportional zu dem Unterschied der erwarteten und beobachteten Häufigkeiten einer Zelle in der zugrundeliegenden Kontingenztafel. 


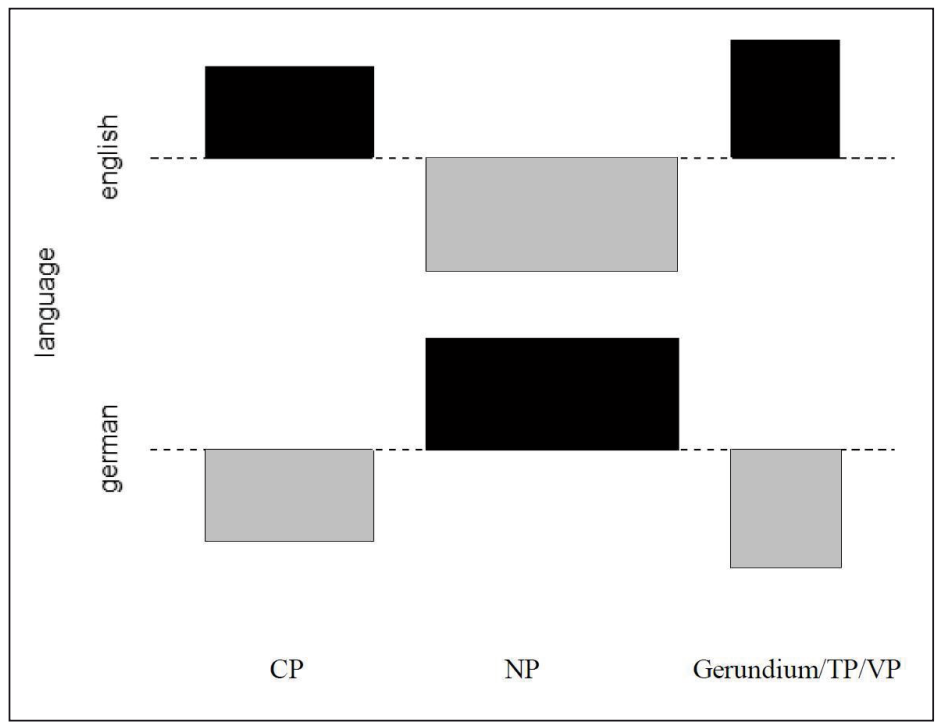

Abb. 4: Assoziationsplot: Syntaktische Kategorie der Cleftkonstituente

Der Assoziationsplot in Abbildung 4 zeigt eine deutliche Asymmetrie zwischen dem Englischen und dem Deutschen: Im Deutschen sind nominale Cleftkonstituenten - absolut und im Vergleich zum Englischen - deutlich überrepräsentiert, während im Englischen satzwertige und verbale Cleftkonstituenten wesentlich häufiger vorkommen als erwartet.

Dieser Befund ist ein zentrales Ergebnis unserer Korpusstudie und es stellt sich die Frage, wie er sich erklären lässt. Diese Frage ist nicht-trivial und stellt letztlich ein multifaktorielles Problem dar. Nach ersten Analysen hat sich gezeigt, dass im Wesentlichen drei Gründe für die in Abbildung 4 erkennbaren Asymmetrien verantwortlich sind:

a) $W h$-Clefts sind im Englischen, nicht aber im Deutschen, syntagmatisch motiviert

Im Englischen ist die Verwendung von $w / h$-Clefts teilweise rein satzintern und syntagmatisch motiviert und erfüllt (im Wesentlichen) dieselbe Funktion wie $i$-Extraposition, nämlich die Rechtsversetzung eines satzwertigen Argumentes (der $w h$-Cleft fungiert gewissermaßen als ,Extrapositionsersatz $)$. (29) ist ein entsprechendes Beispiel. Der $w / h$-Cleft ist hier nicht diskurspragmatisch motiviert und entspricht auch im deutschen Beispiel keinem Cleft (vgl. (30); Deutsch ist hier Originalsprache).

(29) [It is reasonable to suppose that BST could lead to a change in consumption patterns and exacerbate the imbalance in the milk, milk 
products and beef and veal sectors.] What is even more ridiculous is that surpluses are produced even under normal circumstances. (EPEG-6/Ge 1737039)

(30) ... Noch viel unsinniger: Schon unter normalen Bedingungen werden Überschüsse produziert.

Hinzu kommt, dass bei adjektivaler Prädikation in deutschen $w$-Clefts eine Sequenz der Form ... ist, ist, dass ... auftritt, die aus stilistischen Gründen (borror aequi) vermieden wird. Das nicht belegte Beispiel (31) illustriert dieses Problem der, doppelten Kopula'.

(31) Was noch viel unsinniger ist, ist, dass schon unter normalen Bedingungen Überschüsse produziert werden.

b) Wh-Clefts werden zur ,Ausgliederung'von Äußerungskommentaren verwendet

Im Englischen werden wh-Clefts häufig dafür verwendet, ,äußerungskommentierende ${ }^{6}$ Konstituenten vom Rest des Satzes zu trennen. Wir werden diesen Effekt als ,Ebenentrennung' bezeichnen, da der ,Äußerungskommentar' (z.B. What I'm saying is ...) strukturell von der Proposition getrennt wird. (32) ist ein entsprechendes Beispiel. Im Deutschen findet sich in diesen Fällen meistens kein $w$-Cleft und es werden oft eingebettete Verbzweitsätze verwendet (vgl. (33)).

(32) What this means is that we are in a position to continue the European Union's development in line with what is needed. (EPEG-6/Ge 281996)

(33) Das heißt, wir sind in der Lage, die Europäische Union entsprechend den Notwendigkeiten fortzuentwickeln.

c) Das Deutsche verwendet Nominalisierungen, wo das Englische Cleftsätze hat

Im Deutschen finden sich oft Nominalisierungen, sowohl im $w$-Satz als auch in der VP, wo das Englische satzwertige Clefts verwendet. Die Sätze in (34) und (35) bilden ein entsprechendes Paar.

(34) What is crucial, of course, is that we welcome the Member States and that this House - soon to be newly elected - can codecide on the new President of the Commission ... (EPEG-6/Du 348586)

(35) Das Entscheidende ist selbstverständlich, dass wir die Beitrittskandidaten herzlich empfangen ... 
Dieser Kontrast ist auch von Fischer (2009) identifiziert und mit der generell stärker nominalen Syntax des Deutschen erklärt worden. Unsere Daten bestätigen die von Fischer (2009) gemachten Beobachtungen. Im Fall von (35) wäre z.B. eine Nominalisierung im Englischen gar nicht ohne weiteres möglich.

\subsection{Die syntaktische Funktion des $w / h$-Pronomens}

Die den Cleftkonstituenten entsprechenden $w$-Wörter im $w$-Satz haben - sowohl im Deutschen als auch im Englischen - in den meisten Fällen die Funktion eines Subjekts, eines direkten Objekts oder eines Prädikats. ${ }^{6}$ Ein kategorischer Unterschied zwischen Deutsch und Englisch besteht bei der Behandlung von Komplementen innerhalb von Präpositionalphrasen. Im Englischen können Cleftkonstituenten aus einer PP extrahiert werden (vgl. (36)). Im Deutschen ist das - wie auch in anderen vergleichbaren Fällen von Extraktion - bekanntermaßen nicht möglich (vgl. Hawkins 1986; König/ Gast 2009).

(36) What ${ }_{\mathrm{i}}$ we are talking [about $\mathrm{t}_{\mathrm{j}}$ ] here, however, are medium-sized vessels ... (EPEG-6/Ge 585863)

(37) * Was $_{\mathrm{i}}$ wir hier [über $\mathrm{t}_{\mathrm{i}}$ ] sprechen, sind mittelgroße Schiffe ...

Um Komplemente von Präpositionalphrasen abzuspalten, kann sich das Deutsche aber der $w$-Formen von Präpositionaladverbien bedienen. Dabei wird die Präposition mit dem Komplement - innerhalb des Präpositionaladverbs - extrahiert. (36) kann also durch (38) wiedergegeben werden. Im Europarl-Korpus tritt allerdings in diesem Fall eine andere Struktur auf. ${ }^{7}$ (39) ist ein belegtes Beispiel für die Verwendung von Präpositionaladverbien im Deutschen.

(38) Worüber wir hier sprechen, sind mittelgroße Schiffe ...

(39) Aber worauf es ankommt, ist die politische Verantwortung. (EPEG-6/Fr 1486378)

Da Präpositionalphrasen im Hinblick auf ihre syntaktische Funktion heterogen sind und es sich angesichts der verhältnismäßig geringen Zahlen nicht lohnt, zwischen Adjunkten und Komplementen zu unterscheiden, haben wir diese Fälle unter der Kategorie der ,anderen' syntaktischen Funktionen zusammengefasst. Die Häufigkeiten der vier syntaktischen Funktionen in unseren 740er-Stichproben sind in Abbildung 5 gezeigt.

${ }_{6}$ Interessanterweise sind indirekte Objekte in beiden Sprachen ausgeschlossen oder zumindest ungewöhnlich bzw. hochgradig infrequent: *Wem ich es gegeben habe, ist Karl, *Who I gave it to was Charles. Wir danken einer/m anonymen Gutachter/in für diesen Hinweis.

Der entsprechende Satz im Korpus weist Verbzweitsyntax auf: Wir sprechen bier jedoch von mittelgroßen, maximal 12 Meter langen Schiffen ... 


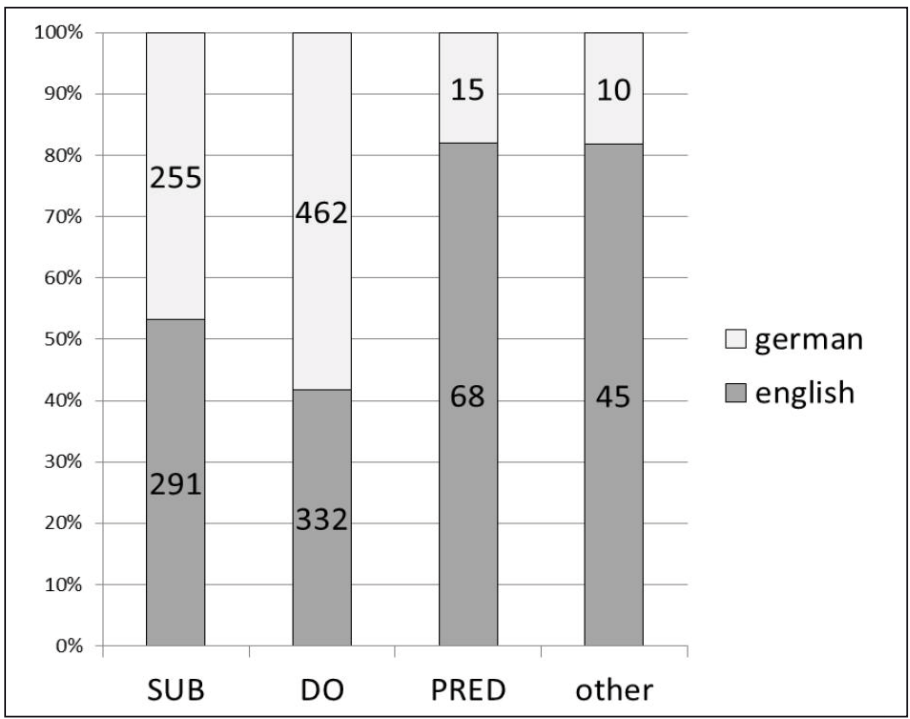

Abb. 5: Syntaktische Funktionen (Häufigkeiten)

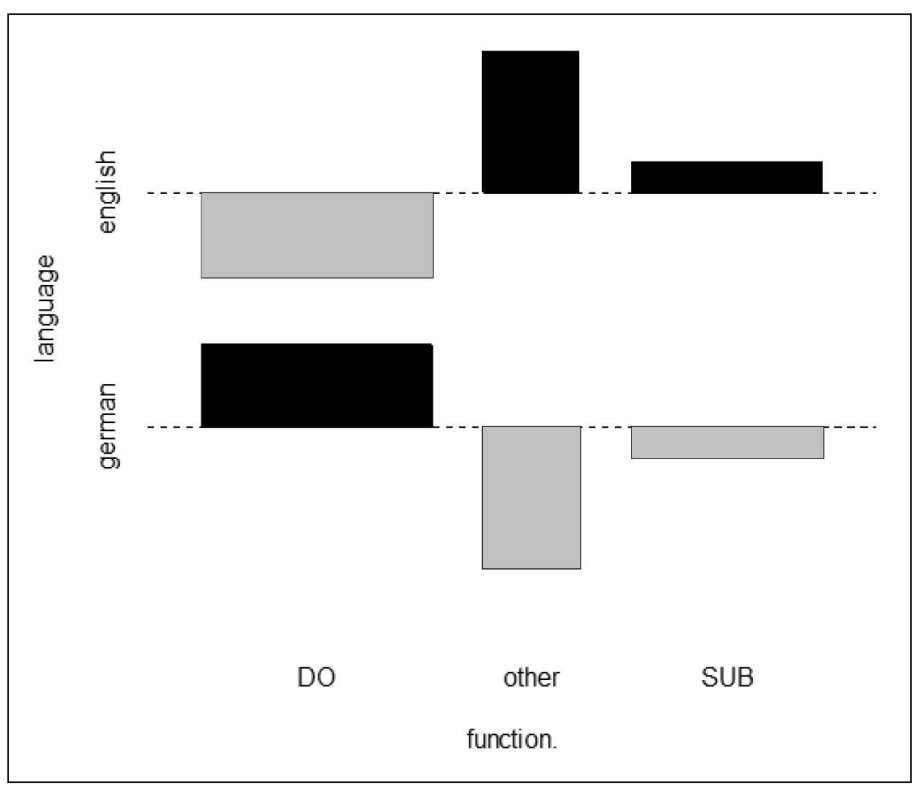

Abb. 6: Syntaktische Funktionen (Assoziationsplot)

Abbildung 6 bildet die Daten in Form eines Assoziationsplots ab. Hier sieht man, dass die Distribution syntaktischer Funktionen (von $w / h$-Pronomen) in $w / h$-Clefts in zweierlei Hinsicht signifikant von der Erwartung bei statis- 
tischer Unabhängigkeit abweicht (Pearson $\mathrm{X}^{2}=79.75$, df $=2, \mathrm{p}<0.001$ ). Der deutlichste Unterschied tritt bei den ,anderen' syntaktischen Funktionen zutage. Das Englische verwendet $w h$-Clefts bei Abspaltung des Komplements einer PP (What I am thinking about is ...) wesentlich häufiger als das Deutsche bei der Abspaltung einer PP durch Verwendung eines Präpositionaladverbs (Woran ich gerade denke, ist ...).

Auch bei den Objekten findet sich eine signifikante Abweichung von statistischer Unabhängigkeit, die allerdings weniger stark ausgeprägt ist als bei den Präpositionalphrasen. Dieser Unterschied ist vermutlich nicht generell und hat unterschiedliche Gründe. Zwar gibt es spezifische Konstruktionen, in denen das Deutsche häufiger als das Englische Objektcleftsätze verwendet. Das trifft insbesondere auf idiomatisierte $w$-Clefts wie Was wir brauchen ... zu (vgl. dazu auch Altmann 2009). Von den 463 Objektcleftsätzen in den deutschen Beispielen enthalten immerhin 197 ( 43\%) die Sequenz was ... braucht/brauchen. In den englischen Entsprechungen hingegen wird in diesen Fällen oft kein Cleftsatz verwendet.

(40) Was wir brauchen, ist mehr Finanzdisziplin ... (EPEG-6/Fr 572983)

(41) Fiscal discipline is required ...

Andererseits haben wir in Abschnitt 4.2 gesehen, dass es Fälle von Objektcleftsätzen gibt, die im Deutschen systematisch vermieden werden, nämlich bei der Ausgliederung eines Äußerungskommentars (What I want to say is ...). Weiterhin dürfte das relative Übergewicht von Objektclefts im Deutschen eine Folge der generellen Seltenheit von satzwertigen Subjektcleftsätzen sein. Wie in Abschnitt 4.2 gesehen, verwendet das Englische - anders als das Deutsche $-w / h$-Clefts oft in Fällen, in denen auch $i t$-Extraposition möglich wäre. Das aus Abbildung 6 ersichtliche, verhältnismäßig schwache Übergewicht der Objektcleftsätze im Deutschen ist also wohl durch verschiedene Faktoren bedingt. In der folgenden Diskussion werden wir uns daher weitgehend auf die Beobachtungen konzentrieren, welche die syntaktische Kategorie der Cleftkonstituente betreffen, insbesondere auf den deutlichen Kontrast zwischen der Häufigkeit von satzwertigen Cleftkonstituenten im Englischen und deren relative Seltenheit im Deutschen.

\section{Zur Motivation von $w / h$-Clefts}

In Abschnitt 4 haben wir quantitative Unterschiede zwischen dem Deutschen und Englischen in der Distribution verschiedener struktureller Cleftsatztypen identifiziert. Wir haben auch schon angedeutet, wie diese Unterschiede zustande kommen. In diesem Abschnitt möchten wir noch einen Schritt weiter gehen und die Frage stellen, welche, allgemeinen Strukturprinzipien' für die beobachteten Kontraste verantwortlich sind. 
Wir werden dafür ein einfaches Modell der Sprachproduktion verwenden, das von Ideen aus dem Bereich der Optimalitätstheorie inspiriert ist, ohne dass wir allerdings mit den,Werkzeugen' der Optimalitätstheorie (z.B. tableaux) arbeiten. Wir gehen davon aus, dass semantisch-pragmatischer, Input ' auf einen strukturellen, Output' abgebildet wird. Grob gesagt, entspricht der Input einer Äußerungsbedeutung und der Output der entsprechenden Äußerung. Die Abbildung von Input auf Output unterliegt bestimmten Bedingungen, die wir ,Output-Bedingungen' nennen. Zu den wichtigsten Output-Bedingungen gehören Regeln der syntaktischen Wohlgeformtheit, die ,absolut ${ }^{\star}$ sind, d.h. ihre Missachtung führt zu Ungrammatikalität. Daneben nehmen wir schwächere, probabilistische Output-Bedingungen an, die gewissermaßen strukturelle Desiderate sind und mit anderen Output-Bedingungen interagieren.

Kontraste zwischen dem Deutschen und Englischen im Bereich der $w / h$-Cleftsatzbildung können wie folgt motiviert werden: Wir nehmen an, dass (idealerweise) in beiden Sprachen derselbe Input vorliegt (d.h. wir unterstellen Übersetzungsäquivalenz zwischen den untersuchten Äußerungen). Außerdem gehen wir davon aus, dass gewisse ,funktionale' Output-Bedingungen in beiden Sprachen identisch sind. Kontraste zwischen dem Deutschen und Englischen treten dann auf, wenn solche Output-Bedingungen auf unterschiedliche Weise erfüllt werden können. Ein wichtiger Unterschied zwischen dem Englischen und Deutschen besteht z.B. darin, dass die ,strukturellen Effekte, die im Englischen bei der Bildung von $w / h$-Clefts auftreten, im Deutschen auch ohne die Bildung eines $w$-Clefts erzielt werden können. Anders ausgedrückt kann das Deutsche bestimmte OutputBedingungen, für deren Erfüllung im Englischen ein wh-Cleft verwendet wird, auch ohne die Bildung eines $w$-Clefts erfüllen.

Wir werden zur genaueren Untersuchung der in Abschnitt 4 beobachteten Kontraste auf vier Output-Bedingungen zurückgreifen: (i) lineare Synchronisierung von Informationsstruktur und Syntax (Abschnitt 5.1), (ii) strukturelle Trennung von Quaestio und Responsio (Abschnitt 5.2), (iii) ,Ebenentrennung, die zur Ausgliederung von Äußerungskommentaren führt (Abschnitt 5.3), und (iv) ,Rechtslastigkeit‘ (Behaghels ,Gesetz der wachsenden Glieder', Abschnitt 5.4).

\subsection{Lineare Synchronisierung von Informationsstruktur und Syntax}

Wie wir in Abschnitt 3 und 4 zu zeigen versucht haben, zeichnen sich $w / h-$ Clefts dadurch aus, dass eine Proposition in zwei Teile aufgespalten wird und dass die beiden Teile diskurspragmatischen Funktionen im Satz entsprechen. Diese Funktionen haben wir ,Quaestio' und ,Responsio' genannt. Durch diese Aufspaltung entsteht ein Effekt, den wir als ,lineare Synchro- 
nisierung von Informationsstruktur und Syntax bezeichnen, und der darin besteht, dass zu Quaestio und Responsio gehöriges Material jeweils adjazent ist. Das lässt sich an folgendem Beispiel zeigen:

(42) What I miss in Mr Martens' report are the guidelines and vision of how a new cooperation could be formed. (EPEG-6/Da 1451517)

(42) kann als, integriertes Adjazenzpaar' betrachtet werden, das sich aus der Quaestio What do I miss in Mr Martens' report? und der Responsio (I miss) the guidelines and vision of how ... zusammensetzt. Das lässt sich auch aus dem unmittelbaren Diskurskontext ablesen:

(43) Mr Martens has put a lot of work into the report, but unfortunately the result is a compromise. A compromise which covers a large number of very significant points, most of which I agree with.

(EPEG-6/Da 1451513)

Der in Rede stehende Bericht wird zunächst (vordergründig) gelobt $-\mathrm{Mr}$ Martens has put a lot of work into the report-wird dann aber als ,Kompromiss" bezeichnet. Hier wird die Frage aufgeworfen, in welcher Hinsicht der Bericht unzureichend ist. Beim Hörer kann also die Quaestio What does the speaker miss in Mr Martens' report? angenommen werden.

In einem einfachen Satz würden Elemente der Quaestio und der Responsio nach den Regeln der englischen Syntax miteinander vermischt. Bei kanonischer V-NP-PP-Abfolge würde die Struktur in (44) entstehen, wo Elemente der Quaestio von einer gestrichelten, solche der Responsio von einer durchgehenden Linie umrahmt sind:

$$
\begin{aligned}
& \text { Quaestio: What do you miss in Mr Martens' report? } \\
& \text { 'I miss? the guidelines and visions 'in Mr Mr Martens' reporti. }
\end{aligned}
$$

Durch die Bildung eines $w$ h-Clefts sind Quaestio und Responsio jeweils adjazent. Diesen Effekt bezeichnen wir als, lineare Synchronisierung von Informationsstruktur und Syntax $:^{6}$

$$
\text { 'W What I miss in Mr Martens' report' are the guidelines and visions. }
$$

Im Deutschen ist in vielen Fällen - wie auch im Fall von (42) - die Bildung eines $w$-Clefts nicht notwendig, um eine, synchrone' Struktur herzustellen, da die Wahlfreiheit bei der Vorfeldbesetzung dieses bereits ermöglicht. (46) ist die deutsche Entsprechung von (45):

$$
\begin{aligned}
& \text { In Mr. Martens Bericht vermisse ich ideshalb die übergreifenden } \\
& \text { Entwicklungslinien... }
\end{aligned}
$$

In diesem Beispiel hätte eine synchrone Struktur auch durch ,heavy NP-shift ${ }^{t}$ hergestellt werden können, also durch eine alternative, ebenfalls nicht-kanonische Wortfolge. 
Die grundlegenden Unterschiede in der Satzstruktur des Deutschen und Englischen - V2 vs. (relativ rigides) SVO - sind zweifellos dafür verantwortlich, dass das Englische in vielen Fällen einen wh-Cleft verwendet, bei denen im Deutschen kanonische Verbzweit-Wortfolge auftritt. Weitere Beispiele aus dem Europarl-Korpus sind in (47) bis (49) gegeben. Die Quaestio ist jeweils gestrichelt umrahmt.

(47) a. 'What are at stake ire cross-border and domestic policy matters of common interest, such as illegal immigration, the sex trade and trafficking in human beings, including women. (EPEG-6/Sv 373313)

b. 'A Âuf dem Ŝ̀iel stehen ' gemeinsame innenpolitische wie grenzüberschreitendē Interessen etwa bezüglich solcher Probleme wie Menschenhandel, Prostitution, Frauenhandel und illegale Einwanderung.

(48) a. TWhat is especially important now is the continued support of the international community and the European Union, for the country faces enormous challenges. (EPEG-6/Ge 199106)

b. 'Ümso notwendiger ist nun id die weitere Unterstützung durch die Völkergemeinschaft und die Europäische Union, denn das Land steht vor enormen Herausforderungen.

(49) a. W'What is perhaps more dangerous is the persistent silence on the overall budgetary implications of transforming, community ${ }^{6} \mathrm{Eu}-$ rope, which herald difficult meetings with the people. (EPEG-6/Fr 232747)

b. 'Noch gefährlicher ist vièlleichtidas hartnäckige Schweigen zu den allgemeinen haushaltspolitischen Auswirkungen der Umgestaltung des gemeinschaftlichen Europa, die der Bevölkerung schwer vermittelbar sein werden.

Im Rahmen unseres Vergleichsmodells können wir sagen, dass die OutputBedingung der linearen Synchronisierung im Deutschen oft ohne Bildung eines Cleftsatzes erfüllt werden kann, während das im Englischen nicht möglich ist. In diesen Fällen liegt im Englischen, nicht aber im Deutschen, eine Motivation für die Verwendung eines Cleftsatzes vor. Dieser Unterschied dürfte (neben anderen Faktoren) maßgeblich für die - im Vergleich zum Englischen - relative Seltenheit von $w$-Clefts im Deutschen sein. Wie wir sehen werden, ist er allerdings nur ein Faktor neben anderen.

\subsection{Strukturelle Trennung von Quaestio und Responsio}

Bisher haben wir Fälle besprochen, in denen im Englischen, nicht aber im Deutschen, ein Cleftsatz motiviert war. Es stellt sich die Frage, warum das 
Deutsche überhaupt wh-Clefts verwendet, wenn es doch die Output-Bedingung der lineare Synchronisierung von Informationsstruktur und Syntax (in den meisten Fällen) auch ohne Cleftsatzbildung erfüllen kann. In diesem Abschnitt werden wir einen Faktor erörtern, der unseres Erachtens eine zentrale Rolle bei der Motivation von Cleftsätzen in beiden untersuchten Sprachen spielt.

Neben der in Abschnitt 5.1 besprochenen, linearen Synchronisierung hat die Bildung von $w / h$-Clefts einen weiteren wichtigen Effekt: Die beiden diskurspragmatischen Funktionen der Quaestio und der Responsio sind in unterschiedlichen Konstituenten ,abgelegt ${ }^{\dagger}$. Diesen Effekt werden wir als ,strukturelle Trennung von Quaestio und Responsio“ bezeichnen. Er lässt sich anhand unseres Dezentralisierungsbeispiels darstellen, wie in (50) gezeigt:

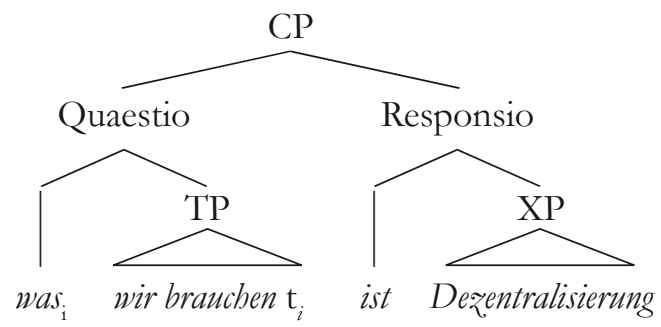

Die strukturelle Trennung von Quaestio und Responsio erlaubt eine genauere Synchronisierung von Prosodie und Syntax als ein einfacher Satz. ${ }^{9} \mathrm{Da}$ Quaestio und Responsio jeweils Konstituenten auf der obersten Ebene des Satzes bilden, können sie vollständig isomorph auf Intonationsphrasen abgebildet werden. Das ist vor allem dann ,hilfreich', wenn beide Komponenten einen Nuklearakzent enthalten, wie in (51) (wir haben zwar keine prosodische Information, die hier angenommene Verteilung von [durch GROSSBUCHSTABEN markierte] Nuklearakzenten scheint uns aber ziemlich eindeutig aus dem Kontext hervorzugehen). Die Quaestio ist wiederum gestrichelt umrahmt.

(51) Das ist ein vernünftiger und richtiger Ansatz ... und wir unterstützen alle Anstrengungen, um die notwendigen Informationssysteme dazu

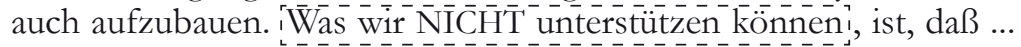
das Europäische Parlament ... außen Vóngelassen wurde. (EPEG-6/Ge 1416964)

Ein(e) Gutachter(in) weist uns völlig zurecht darauf hin, dass eine strikte Zweiteilung in Quaestio und Responsio in manchen Fällen eine Idealisierung darstellt. Insbesondere Satzadverbien sind oft nicht eindeutig einer dieser Komponenten zuzuordnen. Es scheint z.B. keinen großen Unterschied zu machen, ob wahrscheinlich in den folgenden Beispielen im $w$-Satz oder in der Cleftkonstituente steht: Was wir wahrscheinlich brauchen, ist Dezentralisierung oder Was wir brauchen, ist wabrscheinlich Dezentralisierung. Die wahrheitsfunktionale Äquivalenz ergibt sich aus der (prinzipiellen) Reduzierbarkeit von $w / h$-Clefts. Ob und in welcher Weise Satzadverbien in solchen Konstruktionen mit Informationsstruktur interagieren, ist eine interessante Frage, der wir an dieser Stelle leider nicht nachgehen können. 
Die Bildung eines $w / h$-Clefts ist also dadurch motiviert, dass sie - anders als ein einfacher Verbzweitsatz - die Output-Bedingung der ,strukturellen Trennung von Quaestio und Responsio' erfüllt. In einem einfachen Satz können diese Komponenten zwar , linear synchronisiert ${ }^{\natural}$ werden, die Quaestio (und deren IP) entspricht aber keiner eigenen Konstituente:

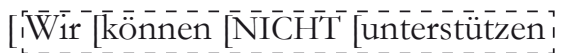

$$
\begin{aligned}
& \text { [ dass das Parlament außen VORgelassen wurde]]]] }
\end{aligned}
$$

Da wir davon ausgehen, dass die in diesem Abschnitt besprochene OutputBedingung in beiden Sprachen gleichermaßen gilt, und dass sie in beiden Sprachen die Bildung eines $w / h$-Clefts motiviert, ist sie nicht für Kontraste, sondern für Gemeinsamkeiten zwischen dem Deutschen und dem Englischen verantwortlich. In der Tat scheinen $w / h$-Clefts im Deutschen - und im Englischen erst recht - oft dann anzutreffen zu sein, wenn sich im Diskurskontext ein mehr oder weniger expliziter Kontrast rekonstruieren lässt. Es finden sich aber auch zahlreiche Fälle, in denen zwar kein Kontrast im Sinne zweier sich gegenseitig ausschließender Optionen vorliegt, sondern lediglich eine paradigmatische Relation zwischen einem Element in der Quaestio und einer vorher erwähnten (hier unterstrichenen) Konstituente besteht. Diese Relation kann z.B. auch die der Identität sein, wie in (53).

(53) Wir können auch die Worte von Mary Robinson heranziehen, die das, was im Zusammenhang mit den Sanktionen geschieht, nachdrücklich verurteilt hat. I' $\overline{\mathrm{W}} \overline{\mathrm{as}}$ geschiehtì, ist, daß Opfer, unschuldige Menschen, Frauen und K' Kinderer, Leute, die absolut machtlos sind, Leute ohne jeden Einfluß verfolgt werden. (EPEG-6/En 77787-8)

Hier wiederholt der $w$-Satz eine Quaestio, die vorher bereits eingeführt wurde. Zunächst wird sehr allgemein das, was im Zusammenhang mit den Sanktionen gescbiebt, erwähnt. Im $w$-Cleft wird es dann wieder aufgegriffen und durch die Cleftkonstituente spezifiziert. Angesichts solcher Beispiele können wir also die Bedingungen, die eine strukturelle Trennung der Proposition in Quaestio und Responsio motivieren, allgemeiner fassen: Es muss eine paradigmatische Beziehung zwischen der Quaestio und einem Element in der unmittelbaren Diskursumgebung vorliegen.

Wie u.a. Birner/Ward (1998) gezeigt haben, besteht eine enge Beziehung zwischen über die Satzgrenze hinausreichenden paradigmatischen Relationen und der Herstellung von Kohäsion (im Sinne von Halliday/Hasan 1976). In der Tat haben $w / h$-Clefts, die durch solche paradigmatischen Bedingungen motiviert sind, oft eine kohäsive Funktion. In (53) z.B. wird im Kontext gegebene Information zunächst wiederholt und dann, elaboriert ${ }^{\star}$.

Die von $w / h$-Clefts herbeigeführte strukturelle Trennung von Quaestio und Responsio macht paradigmatische Relationen zwischen Elementen in der Quaestio und im Diskurskontext transparent. Sie kann prosodisch oder 
auch semantisch (textuell) motiviert sein. Im Vergleich zur in Abschnitt 5.1 besprochenen linearen - also syntagmatischen - Synchronisierung können $w / h$-Clefts also auch der , paradigmatischen Synchronisierung ${ }^{6}$ dienen. Eine erste Generalisierung, die sich nun abzeichnet, besteht darin, dass deutsche $w$-Clefts überwiegend paradigmatisch motiviert sind, während englische whClefts oft auch eine rein syntagmatische Funktion erfüllen.

\subsection{Ebenentrennung und Ausgliederung von Äußerungskommentaren}

Die für $w / h$-Clefts charakteristische strukturelle, Zweiteilung' eines Satzes kann auch satzsemantisch motiviert sein. Oft hat die im $w / h$-Satz enthaltene Information kommentierenden Charakter und trägt keine Information zur ausgedrückten Proposition bei. (54) ist ein entsprechendes Beispiel aus dem Englischen:

(54) What this means is that we are in a position to continue the European Union's development in line with what is needed.

(EPEG-6/Ge 281996)

Der propositionale Gehalt des $w / h$-Clefts in (54) ist im Wesentlichen, We are in a position to continue ...', und der wh-Satz What this means ... enthält nur eine Kontextualisierung bzw. einen, Äußerungskommentar'. Der Effekt der Cleftsatzbildung besteht also in diesem Fall darin, dass die ,eigentliche Proposition strukturell (auf der höchsten Ebene des Satzes) vom Äußerungskommentar getrennt wird. Allgemein gesprochen, werden die propositionale und die illokutive Ebene des Satzes getrennt. Wir bezeichnen das dieser Operation zugrundeliegende Prinzip daher als ,Ebenentrennung.

Im Deutschen finden sich Cleftsätze mit Äußerungskommentaren im $w$-Satz nur sehr selten. Stattdessen werden an den entsprechenden Korpusstellen typischerweise eingebettete Verbzweitsätze verwendet, wie auch in der deutschen Version der englischen Textstelle in (54):

(55) Das heißt, wir sind in der Lage, die Europäische Union entsprechend den Notwendigkeiten fortzuentwickeln.

In anderen Fällen verwendet das Deutsche Parenthesen:

We did not succeed in that, but it does not matter. What I would like to say is that I am very glad that the Greens' Amendment No 25 was rejected. (EPEG-6/Ge 353419)

Ich bin sehr froh - und das möchte ich auch erwähnen -, dass der Antrag 25 der Grünen abgelehnt wurde.

Wie bereits erwähnt wurde, scheinen die kommentierenden wh-Clefts des Englischen in erster Linie satzsemantisch motiviert zu sein. Sie dienen gewissermaßen dazu, das, illokutive Hauptgewicht' des Satzes in die zweite 
Satzhälfte zu legen. Der $w h$-Satz ist präsupponiert und daher für den propositionalen Gehalt des Satzes weitgehend irrelevant. Das Deutsche erzielt diesen Effekt auf eine andere Weise: Indem der eingebettete Satz ,Hauptsatzsyntax ${ }^{6}$ aufweist, wird er gegenüber dem übergeordneten Prädikat gewissermaßen , aufgewertet'.

Der in diesem Abschnitt beschriebene Unterschied ist u.a. dafür verantwortlich, dass das Deutsche wesentlich weniger satzwertige $w / h$-Clefts verwendet als das Englische. Die Output-Bedingung der Ebenentrennung kann im Deutschen auch ohne die Bildung eines $w / h$-Clefts erfüllt werden. Im Englischen steht keine den eingebetteten Verbzweitsätzen entsprechende Struktur zur Verfügung. Man kann zwar mit bestimmten Prädikaten eingebettete Sätze ohne Subjunktion bilden (I think this is a good idea), diese sind aber nichtsdestotrotz syntaktisch wie semantisch untergeordnet.

\subsection{Rechtslastigkeit}

Während die bisher behandelten Motivationen für Cleftsatzbildung in der einen oder anderen Weise ,funktional' - diskurspragmatisch oder satzsemantisch - motiviert waren, scheint in den in diesem Abschnitt besprochenen Fällen eine solche Motivation untergeordnet zu sein oder auch gar nicht vorzuliegen. (58) ist ein entsprechendes Beispiel:

(58) The first version of the report was used as a chance for them to get on their hobbyhorse and oppose nuclear energy. What is remarkable is that there was no mention, whatsoever, in the report of the Commission's action programme, which contains many sensible initiatives. (EPEG-6/Fr 119772)

Der Kontext suggeriert nicht, dass eine Quaestio der Form What is remarkable? zur Diskussion steht. Der Cleftsatz scheint vielmehr die Funktion zu erfüllen, das satzwertige Subjekt that there was no mention ... in eine finale Position zu rücken. Mit anderen Worten, der $w / h$-Cleft erfüllt mehr oder weniger dieselbe Funktion wie it-Extraposition, und in der Tat ist der textuelle Effekt zwischen (58) und dem entsprechenden Satz mit Extraposition minimal:

(59) The first version of the report was used as a chance for them to get on their hobbyhorse and oppose nuclear energy. It is remarkable that there was no mention, whatsoever, in the report of the Commission's action programme, which contains many sensible initiatives.

Fälle wie (58) haben wir in Abschnitt 4.2 mit dem Begriff des ,Extrapositionsersatzes" charakterisiert. Wie eingangs erwähnt, betrachten wir diese Verwendungen gewissermaßen als ,zweckentfremdet‘. Die ursprüngliche, diskurspragmatische Funktion des Cleftsatzes wird von einer rein syntak- 
tischen Funktion in den Hintergrund gedrängt. Diese Cleftsätze sind durch Behaghels, Gesetz der wachsenden Glieder (bzw. entsprechende Prinzipien wie die von Hawkins 1994, 2004 postulierten) motiviert. Wir werden der Einfachheit halber von ,Rechtslastigkeit' sprechen. Die Output-Bedingung der ,Rechtslastigkeit‘ besagt, dass von zwei Schwesterkonstituenten die rechte immer ,schwerer' sein sollte als die linke. Der strukturelle Unterschied zwischen dem wh-Cleft und kanonischer SVO-Wortfolge ist in (60) und (61) gezeigt.

(60) Rechtslastige Struktur

[What is remarkable] [is [that there was no mention ...]]

(61) Linkslastige Struktur

[That there was no mention ...] [is [remarkable]]

Da im Deutschen satzwertige Subjekte auch ohne Verwendung eines $w$ Clefts in satzfinaler Position stehen können, ist es nicht verwunderlich, dass die überwiegende Mehrzahl der englischen $w$ h-Clefts, die durch die OutputBedingung der Rechtslastigkeit motiviert sind, im Deutschen nicht durch einen $w$-Cleft wiedergegeben werden. Die folgenden Beispiele illustrieren dies.

(62) a. 'What must be really underlined is that, in my view, we must firmly support a Commission proposal which, essentially with regard to an issue as serious as the budgets of the Member States, is inclined to give priority to the revenue actually collected rather than criteria such as the amounts due in the field of taxes and social contributions. (EPEG-6/Sp 41184)

b. 'W nach ein Vorschlag der Kommission, der in erster Linie und bei einem so wichtigen Thema wie den Haushaltsplänen der Mitgliedstaaten darauf gerichtet ist, den tatsächlich vereinnahmten Beträgen Vorrang einzuräumen vor Kriterien wie den auf Veranlagungen beruhenden Beträgen von Steuern und Sozialbeiträgen, entschieden unterstützt werden muss.

(63) a. What is perhaps most remarkable of all, however, is how little all this is discussed by the Bank's owners, in the Member States and in the parliaments of the various Member States which, I believe, are in many cases quite unaware of the Bank's activities. (EPEG-6/Sv 240125)

b. AAm erstaunlichsten ist aber vielleicht keit dieses Thema bei den Eigentümern der Bank, den Mitgliedstaaten, und in den nationalen Parlamenten findet, die, so glaube ich, in vielen Fällen nur wenig Kenntnis über die Tätigkeit der EIB haben. 
(64) a. 'What is apparent thoughi is that our own market economy is not yet performing to optimum effect. (EPEG-6/Du 344183)

b. IA Allerdings hat sich gezeigt, dass unsere eigene Marktwirtschaft noch nicht optimal funktioniert.

Der in diesem Abschnitt behandelte Unterschied ist maßgeblich dafür verantwortlich, dass $w / h$-Subjektclefts mit satzwertiger Cleftkonstituente im Englischen wesentlich häufiger sind als im Deutschen. Obwohl auch die entsprechenden Objektclefts ein deutliches Ungleichgewicht zugunsten des Englischen aufweisen, ist dieser Kontrast bei den Subjektclefts noch ausgeprägter: Während 15 von 143 Objektclefts mit satzwertiger Cleftkonstituente aus der englischen 740er-Stichprobe auch im Deutschen einem $w$ Cleft entsprechen (ca. 10\%), sind von 127 Subjektclefts mit satzwertiger Konstituente nur drei im Deutschen auch als $w$-Cleft wiedergegeben (ca. $2 \%$ ).

\subsection{Zusammenfassung}

Wir haben vier Faktoren unterschieden, welche die Bildung von $w / h$-Clefts zu begünstigen scheinen und insofern als ,Motivationen' für deren Verwendung betrachtet werden können: (i) lineare Synchronisierung von Informationsstruktur und Syntax, (ii) strukturelle Trennung von Quaestio und Responsio, (iii) Ebenentrennung (Ausgliederung von Äußerungskommentaren), und (iv) das strukturelle Desiderat der Rechtslastigkeit. Wie wir gesehen haben, sind drei dieser Motivationen vor allem im Englischen für die Bildung von wh-Clefts verantwortlich, nämlich (i), (iii) und (iv). Im Deutschen sind $w / h$-Clefts typischerweise durch eine paradigmatische Relation motiviert, die zwischen einem Element in der Quaestio und der Diskursumgebung besteht, was wiederum die (probabilistische) Output-Bedingung der strukturellen Trennung von Quaestio und Responsio zur Folge hat. Die vier besprochenen Motivationen für $w h$-Clefts im Englischen und deren typische Entsprechungen sind in Tabelle 2 zusammengefasst.

\begin{tabular}{|l|l|l|}
\hline & Englisch & Deutsch \\
\hline lineare Synchronisierung & $w h$-Cleft & V2-Satz \\
\hline $\begin{array}{l}\text { strukturelle Trennung } \\
\text { (Quaestio/Responsio) }\end{array}$ & $w h$-Cleft & $w$-Cleft \\
\hline $\begin{array}{l}\text { Ebenentrennung } \\
\text { (Kommentar/Proposition) }\end{array}$ & $w h$-Cleft & eingebettete V2-Sätze \\
\hline Rechtslastigkeit & $w h$-Cleft & V2-Satz \\
\hline
\end{tabular}

Tab. 2: Vier Motivationen für die Bildung von $w / h$-Clefts im Englischen und Deutschen 


\section{Fazit}

Ein Vergleich von probabilistisch distribuierten Strukturen wie $w / h$-Clefts stellt die kontrastive Linguistik vor nicht-triviale Herausforderungen. Da eine einheitliche funktionale Motivation der Verwendung solcher Strukturen nicht einfach zu ermitteln ist, ist ein ,klassischer', onomasiologischer Ansatz nicht fruchtbar. Wir sind daher weitgehend semasiologisch vorgegangen und haben die untersuchten strukturellen Konfigurationen selbst als Grundlage des Vergleichs verwendet. In einem zweiten Schritt haben wir dann Motivationen für die Verwendung der entsprechenden Strukturen ermittelt. Die Identifikation der Motivationen war dabei von der uns zur Verfügung stehenden quantitativ-kontrastiven Evidenz geleitet. Wir haben uns auf die Faktoren konzentriert, von denen wir uns Aufschluss über die ermittelten quantitativen Kontraste erhofften.

Im Rahmen eines ,kontrastiven Vergleichsmodells' haben wir dann Output-Bedingungen identifiziert, die $w / h$-Clefts zu motivieren scheinen. Dabei hat sich gezeigt, dass zahlreiche englische wh-Clefts durch Bedingungen motiviert sind, die im Deutschen auch durch Verwendung kanonischer Wortfolgen erfüllt werden können. In diesen Fällen wird erwartet, dass die Verwendung eines $w$-Clefts im Deutschen signifikant seltener ist als im Englischen. Die in Abschnitt 5 vorgeschlagenen Generalisierungen müssen allerdings noch durch genauere quantitative Untersuchungen gestützt werden, z.B. indem die Stichprobe nach funktionalen Kriterien wie den in Abschnitt 5 besprochenen kodiert werden.

Als vorläufiges Ergebnis können wir festhalten, dass $w$-Clefts im Deutschen überwiegend diskurspragmatisch und paradigmatisch motiviert sind (vgl. Abschnitt 5.2), während sie im Englischen auch eine satzsemantische und syntagmatische Funktion erfüllen. Aus historischer Sicht legt die Distribution von $w / h$-Clefts im Englischen und Deutschen nahe, dass die deutschen Daten den ,funktionalen Kernbereich' der $w / h$-Clefts abdecken, während sich im Englischen auch (durch Exaptation), zweckentfremdete' Verwendungen finden. Auch zur Überprüfung dieser Hypothese sind weitere detaillierte Untersuchungen notwendig.

\section{Literatur}

Akmajian, Adrian (1970): On deriving cleft sentences from pseudo-cleft sentences. In: Linguistic Inquiry 1, S. 149-168.

Altmann, Hans (1981): Formen der Herausstellung im Deutschen: Rechtsversetzung, Linksversetzung, freies Thema und verwandte Konstruktionen. (= Linguistische Arbeiten 106). Tübingen. 
Altmann, Hans (2009): Clefts (Spaltsätze) und Pseudoclefts (Sperrsätze) im Deutschen. In: Brdar-Szabó, Rita/Knipf-Komlósi, Erzsébet/Péteri, Attila (Hg.): An der Grenze zwischen Grammatik und Pragmatik. (= Deutsche Sprachwissenschaft international 3). Frankfurt a.M. u.a., S. 13-34.

Biber, Douglas (1993): Representativeness in corpus design. In: Literary and Linguistic Computing 8, S. 243-257.

Birner, Betty J./Ward, Gregory (1998): Information status and noncanonical word order in English. (= Studies in Language Companion Series 40). Amsterdam/Philadelphia.

Büring, Daniel (2003): On D-Trees, Beans, and B-Accents. In: Linguistics and Philosophy 26, S. 511-545.

Carlson, Lauri (1983): Dialogue games. An approach to discourse analysis. (= Synthese Language Library 17). Dordrecht u.a.

den Dikken, Marcel (2009): Predication and specification in the syntax of cleft sentences. Ms., CUNY Graduate Center. New York.

Duden (2009): Der Duden in zwölf Bänden. Bd. 4: Die Grammatik - unentbehrlich für richtiges Deutsch. Hrsg. v. d. Dudenredaktion. 8., überarb. Aufl. Mannheim u.a.

Faraci, Robert (1971): The deep question of pseudo-clefts. In: English Linguistics 6, S. $48-85$.

Fischer, Klaus (2009): Cleft sentences: form, function, and translation. In: Journal of Germanic Linguistics 21.2, S. 167-191.

Friendly, Michael (1992): Graphical methods for categorical data. In: SAS User Group International Conference Proceedings 17, S. 1367-1373.

Gast, Volker (i.Ersch.): Contrastive analysis: Theories and Methods. In: Kortmann, Bernd/Kabatek, Johannes (Hg.): Dictionaries of linguistics and communication science: linguistic theory and methodology. Berlin.

Gast, Volker/van der Auwera, Johan (2011): Scalar additive operators in the languages of Europe. In: Language 87.1, S. 2-54.

Halliday, Michael Alexander Kirkwood/Hasan, Ruqayia (1976): Cohesion in English. (= English Language Series 9). London.

Haspelmath, Martin (2010): Comparative concepts and descriptive categories. In: Language 86.3 , S. 663-687.

Hawkins, John (1986): A comparative typology of English and German: unifying the contrasts. London u.a.

Hawkins, John (1994): A performance theory of order and constituency. (= Cambridge Studies in Linguistics 73). Cambridge u.a.

Hawkins, John (2004): Efficiency and complexity in Grammars. Oxford u.a.

Hintikka, Jaakko (1973): Logic, language-games and information: Kantian themes in the philosophy of logic. Oxford.

Huddleston, Rodney/Pullum, Geoffrey (2002): The Cambridge grammar of the English language. Cambridge u.a. 
Johansson, Stig (2000): Contrastive linguistics and corpora. Reports from the project ,Languages in Contrast‘. Oslo.

Klein, Wolfgang/Stutterheim, Christiane von (1987): Quaestio und referentielle Bewegung in Erzählungen. In: Linguistische Berichte 109, S. 163-183.

Klein, Wolfgang/Stutterheim, Christiane von (1992): Textstruktur und referentielle Bewegung. In: Zeitschrift für Literaturwissenschaft und Linguistik 86, S. 67-92.

Klima, Edward S./Bellugi-Klima, Ursula (1969): Syntactic regularities in the speech of children. In: Reibel, David A./Schane, Sanford A. (Hg.): Modern Studies in English. Englewood Cliffs, NJ, S. 448-466.

Koehn, Philipp (2005): Europarl: a parallel corpus for statistical machine translation. MT Summit X, Phuket, S. 79-86.

König, Ekkehard/Gast, Volker (2009): Understanding English-German contrasts. 2., neu bearb. Aufl. (= Grundlagen der Anglistik und Amerikanistik 29). Berlin.

Lass, Roger (1990): How to do things with junk: exaptation in language evolution. In: Journal of Linguistics 26, S. 79-102.

Lewis, David (1969): Convention. A philosophical study. Cambridge, MA.

Prince, Ellen F. (1978): A comparison of $w h$-clefts and it-clefts in discourse. In: Language 54, S. 883-906.

Roberts, Craige (1996): Information structure in discourse: towards an integrated formal theory of pragmatics. In: Yoon, Jae-Hak/Kathol, Andreas (Hg.): Papers in Semantics. (= OSU Working Papers in Linguistics 49). Columbus, OH, S. 91-136.

Roberts, Craige (2004): Context in dynamic interpretation. In: Horn, Laurence/Ward, Gregory (Hg.): Handbook of contemporary pragmatic theory. London, S. 197-220.

Ross, John Robert (1967): Constraints on variables in syntax. Doctoral dissertation, Massachusetts Institute of Technology. Internet: http://hdl.handle.net/1721.1/15166 (Stand: 26.09.2011).

Ross, John Robert (2011): Ein grüner Faden. Gequatsche zwischen Pseudos und Haj 1964. Handout of a talk given at the Free University Berlin, 11 January, 2011. 